\title{
Gut metagenomes of type 2 diabetic patients have characteristic single- nucleotide polymorphism distribution in Bacteroides coprocola
}

Yaowen Chen ${ }^{1}$, Zongcheng $\mathrm{Li}^{1}$, Shuofeng $\mathrm{Hu}^{1}$, Jian Zhang ${ }^{1}$, Jiaqi $\mathrm{Wu}^{1}$, Ningsheng Shao ${ }^{1}$, Xiaochen $\mathrm{Bo}^{2}$, Ming $\mathrm{Ni}^{2^{*}}$ and Xiaomin Ying ${ }^{1 *}$ (D)

\begin{abstract}
Background: Gut microbes play a critical role in human health and disease, and researchers have begun to characterize their genomes, the so-called gut metagenome. Thus far, metagenomics studies have focused on genus- or species-level composition and microbial gene sets, while strain-level composition and single-nucleotide polymorphism (SNP) have been overlooked. The gut metagenomes of type 2 diabetes (T2D) patients have been found to be enriched with butyrate-producing bacteria and sulfate reduction functions. However, it is not known whether the gut metagenomes of T2D patients have characteristic strain patterns or SNP distributions.

Findings: We downloaded public gut metagenome datasets from 170 T2D patients and 174 healthy controls and performed a systematic comparative analysis of their metagenome SNPs. We found that Bacteroides coprocola, whose relative abundance did not differ between the groups, had a characteristic distribution of SNPs in the T2D patient group. We identified 65 genes, all in B. coprocola, that had remarkably different enrichment of SNPs. The first and sixth ranked genes encode glycosyl hydrolases (GenBank accession EDU99824.1 and EDV02301.1). Interestingly, alpha-glucosidase, which is also a glycosyl hydrolase located in the intestine, is an important drug target of T2D. These results suggest that different strains of $B$. coprocola may have different roles in human gut and a specific set of $B$. coprocola strains are correlated with T2D.
\end{abstract}

Keywords: Type 2 diabetes, Metagenome, Bacteria, Phylogenetic analysis, SNP enrichment

\section{Background}

Human gut microbiota are critical to human health and have been related to various disease conditions, such as obesity [1], diabetes [2-4], cirrhosis of the liver [5], inflammatory bowel disease [6], atopic dermatitis [7], and pulmonary inflammation [8]. Next-generation sequencing (NGS) and bioinformatics technologies provide access to the genetic information of the entire microbiome and thus enable systematic investigation of its composition and functional genetics.

\footnotetext{
* Correspondence: ni.ming@163.com; yingxmbio@gmail.com

${ }^{2}$ Beijing Institute of Radiation Medicine, Beijing 100850, People's Republic of China

'Beijing Institute of Basic Medical Sciences, Beijing 100850, People's Republic of China
}

(c) The Author(s). 2017 Open Access This article is distributed under the terms of the Creative Commons Attribution 4.0 International License (http://creativecommons.org/licenses/by/4.0/), which permits unrestricted use, distribution, and reproduction in any medium, provided you give appropriate credit to the original author(s) and the source, provide a link to the Creative Commons license, and indicate if changes were made. The Creative Commons Public Domain Dedication waiver (http://creativecommons.org/publicdomain/zero/1.0/) applies to the data made available in this article, unless otherwise stated. the relative abundance of bacterial species or pathway enrichment between patients and healthy controls (HCs). However, little work has been done to elucidate strain-level variations or single-nucleotide polymorphisms (SNPs) in the metagenome. Because even slight nucleotide variations can alter the pathogenic behavior and antibiotic resistance of bacteria [9-11], analyses of genomic variations (i.e., SNPs, insertions, and deletions) and structural variation of the metagenome are important for understanding microbiome biology.

Regarding strain-level variation in the microbial metagenome, Schloissnig and colleagues proposed a workflow for analyzing metagenomics datasets at the strain level and described the genomic variation landscape of human 
gut microbial genomes [12]. Subsequently, Greenblum et al. detected extensive variations in strain-level copynumbers in the human gut microbiome [13] and Zhu et al. reported considerable differences (based on gene deletions) in the gene content of strains within the same species in the human gut [14]. Tropism and persistence of different oral Neisseria strains have also been studied using metagenomics sequencing [15]. Although genomic variation of microbiomes is well documented by these studies, association of strain-level metagenomics findings with human diseases has been limited.

Type 2 diabetes (T2D) is a complex metabolic disorder afflicting hundreds of millions of people worldwide [16]. Because T2D is related to diet and digestion, the role of gut microbiota in T2D initiation and progression is of great interest. Previous large-scale T2D-related metagenomics research has shown that the proportion of phylum Firmicutes and class Clostridia cells in the microbiome is significantly reduced in T2D [2] and that the gut microbiota of T2D patients tends to have fewer butyrate-producing bacteria, such as Roseburia intestinalis and Faecalibacterium prausnitzii [3].

To the best of our knowledge, no prior study has resolved the association between $\mathrm{T} 2 \mathrm{D}$ and the gut microbiome at a strain or SNP level. Here, we utilized a public NGS dataset resource and performed a comparative study examining the SNPs of gut metagenomes in T2D patients relative to $\mathrm{HCs}$.

\section{Methods}

\section{T2D and control dataset}

Raw NGS datasets of DNA obtained from fecal samples of $170 \mathrm{~T} 2 \mathrm{D}$ patients and 174 HCs in China [3] were downloaded from the NCBI Sequence Read Archive (total data, 1.2 terabases; average sample size, 3.5 gigabases; accession numbers SRA045646 and SRA050230). To reduce SNP false positives, we trimmed abnormal bases and filtered low-quality reads as described in detail previously [12]. For each base (A, T, G and C), a mean number of base calls $(f)$ across all sites and a standard deviation (SD) were calculated. Starting from the first site at $5^{\prime}$ end, the site was trimmed if the base call number for a base was beyond $f \pm 2 \times \mathrm{SD}$. The trimming at $5^{\prime}$ end was terminated until encountering a site with all base call numbers within the range. Next, Trimmomatic [17] was used to remove adapters and trim low-quality bases $(<Q 20)$ at the $3^{\prime}$ end (parameters: -phred33 ILLUMINACLIP:adapters.fa:1:0:7 TRAILING:20 SLIDINGWINDOW:5:10 MINLEN:45 AVGQUAL:20). A total of 1.04 terabases of data remained after quality control procedures were completed. Clinical information and other characteristics of the 344 individuals included in the analysis were obtained from [3] and [18].

\section{Bacterial reference genome determination}

We first determined a set of bacterial reference genomes for samples included in this study. Metaphlan2, which is based on approximately 17,000 reference genomes [19], was employed to profile the bacterial species in each sample. Genomes of species that were identified by metaphlan 2 in at least four samples were included in the reference set for the alignment analyses. The genome sequences of all of these species were downloaded from the NCBI assembly database and are listed in Additional file 1: Table S1.

\section{Selecting species and genes with sufficient supporting reads}

Clean reads were aligned to the whole reference set with Burrows-Wheeler Aligner-maximal exact match (BWAMEM) [20] in default settings, and only unique alignments were outputted. Species with sufficient supporting reads were selected with a cutoff of $\geq 40 \%$ of the reference genome being covered by a $\geq 10 \times$ depth in at least 20 samples in both the T2D and the control groups. For genes, the cutoff was $\geq 80 \%$ gene sites with $\geq 10 \times$ depth in at least 10 samples in each group. Only the species and genes that met these criteria were subjected to subsequent SNP analyses.

\section{SNP and intra-sample variation calling and filtering}

Two tools, BCFtools [21] and VarScan2 [22], were applied to identify SNPs of the metagenome. The alignment of duplicates by BWA-MEM was first marked and filtered in Picard [23]. Then, SAMtools [24] was used to generate "mpileup" files from the SAM-formatted alignment files. The mpileup files were employed as input files for both BCFtools and VarScan2. The parameters used for BCFtools and VarScan2 were "-vmO z $-\mathrm{V}$ indels" and "pileup2snp min-coverage $10, p$ value 0.05 , min-avg-qual 15 ," respectively. SNPs detected by both the tools were selected. SNPs were further filtered with the requirements of $\mathrm{a} \geq 0.5$ mutated allele (relative to that in the reference genome) frequency, $\geq 4 \times$ supporting reads for the variant, and strand bias of sequencing bases less than tenfold in both BCFtools and VarScan2. Namely, when genome sites with heterozygosity were found in a sample, the major allele was used for the SNP analysis.

To address the heterozygosity or intra-sample variations, mutated allele frequencies (MuAFs) of variant sites were obtained for analysis under polyclonal scenario. The MuAFs of sites were defined as the mean values of outputs by BCFtools and VarScan2, which were highly correlated $\left(R^{2}=0.997\right)$. To examine whether intra-sample variations affect the result, SNPs with a $>0.8 \mathrm{MuAF}$ were selected for a parallel SNP analysis.

\section{Annotation of genes and SNPs}

Gene ontology (GO) annotations of each genome were downloaded from Uniprot [25]. SNPs were annotated in 
SnpEff [26] with the -eff parameter. The genome annotation files used by SnpEff were obtained from GenBank.

\section{Phylogenetic tree construction based on whole-genome level SNPs of B. coprocola}

Based on the reference genome of B. coprocola (reference strain DSM 17136, GenBank accession GCA_0001 54845.1 ), genome regions with $>20 \%$ samples not having valid coverage $(\geq 10 \times$ depth) were discarded. If $\leq 20 \%$ of the samples had invalid coverage in a region, the bases in that region in those samples were labeled as "N." Then, the nucleotides at SNP sites from the samples were extracted to generate a pileup file. Phylogenetic trees were constructed based on the whole-genome level aligned SNPs by using randomized axelerated maximum likelihood (RAxML) v8.2.9 (100 bootstrap replicates), with GTR model of nucleotide substitution, $\gamma$-distributed rates among sites, and Felsenstein correction for ascertainment bias $[27,28]$. The parameters for RAxML were “-\#100 -m ASC_GTRGAMMA -f a.” Rooting was undertaken by using RAxML with “-f I" option. Trees were drawn with the R package ggtree [29].

\section{Phylogenetic tree construction of genes}

The nucleotide sequences of genes were obtained by aligning reads to corresponding full-length genes of the reference genome. For a given gene, gene regions were discarded if with $>20 \%$ samples not having valid coverage ( $\geq 10 \times$ depth) and the coverages of full-length genes are listed in Additional file 1: Table S4. If $\leq 20 \%$ of the samples had invalid coverage in a region, the bases in that region in those samples were labeled as "N." Then, the full-length genes, except the discarded regions, were aligned. The phylogenetic trees of genes were constructed by using RAxML v8.2.9 with settings as these for whole-genome level, but without ascertainment correction. The two clusters are separated at the root of the phylogenetic tree.

\section{Clustering of samples by mutated allele frequencies of sites}

For a given samples, the sites of bacterial genome and gene with a $>0.2 \mathrm{MuAFs}$ were selected. For all samples, we obtained a matrix with the column denoting site positions and the rows denoting samples. If there was no variation or MuAFs <0.2, the MuAFs were set to 0 . Then, hierarchical clustering and affinity propagation (AP) clustering [30] were applied to the matrix. The hclust function of stat package in R v3.3.0 was used for hierarchical clustering with parameters "method = complete," and the output tree was drawn by ggtree. For AP clustering, APcluster in R v3.3.0 was employed with parameters " $K=$ 2." The clusters generated by AP clustering were visualized by using Cytoscape v3.5.0 [31]. For each node, the top five edges connecting the nodes with the largest similarity are shown.

\section{Statistical analysis}

Relative abundance of species and genome/gene densities were compared between the T2D group and control group with the Mann-Whitney test. Fisher's exact test was performed to test for bias of SNP sites and sample enrichment inferred from the phylogenetic tree and clusters by AP clustering. Hypergeometric test was applied for one-tailed test for enrichment of biased SNP sites at gene level. The $q$ value with Storey and Tibshirani's method ( $\mathrm{R}$ package qvalue v1.43.0) was applied for multiple testing correction [21] to identify species and genes with significantly biased SNP distribution.

\section{Results}

Based on gut metagenomics data from 344 individuals (170 T2D patients and 174 healthy controls), we identified a total of 356 bacterial species (Additional file 1: Table S1; Additional file 2: Figure S3) and their relative abundances. Consistent with previous reports, we found that, relative to the $\mathrm{HC}$ group, the T2D group had lower proportions of phylum Firmicutes, class Clostridia, and butyrateproducing bacteria (Additional file 2: Figure S1) [2, 3]. Relative abundance did not differ between the groups for 270 of the 356 species (75.8\%) analyzed (Mann-Whitney test, $p>0.05$; Additional file 1: Table S2).

We selected 20 bacterial species with sufficiently supporting NGS reads in a sufficient number of samples (see "Methods" section, Additional file 1: Table S3) for analysis of SNP distribution. Based on the reference genomes of these 20 species, a total of 5.94 million SNPs were identified, of which $99.65 \%$ were bi-allelic and $0.35 \%$ were tri-allelic. The distributions within these 20 species of the normalized bi-allelic SNP densities calculated for genome regions with valid coverage $(>10 \times)$ in the T2D and $\mathrm{HC}$ groups are reported in Additional file 2: Figure S2. The SNP density distribution differed significantly between the T2D and HC groups (Mann-Whitney test, $p=0.0083, q=0.0258$ ) for only one of the 20 species, namely, Bacteroides coprocola (reference strain DSM 17136, Genbank accession GCA_000154845.1). However, the mean relative abundance of $B$. coprocola for the T2D group $(9.10 \pm 7.09 \%)$ was similar to that for the control group $(8.91 \pm 7.16 \%$; Mann-Whitney test, $p=0.9646)$ in the samples with sufficient reads. Moreover, B. coprocola was prevalent and identified in $31.98 \%(110 / 344)$ of all the samples, ranked the top 24th among the 356 species.

A phylogenetic tree of the samples that was constructed based on the B. coprocola SNPs revealed a biased distribution of T2D patients versus HCs (Fig. 1). The intra-tree distance (quantified based on average 


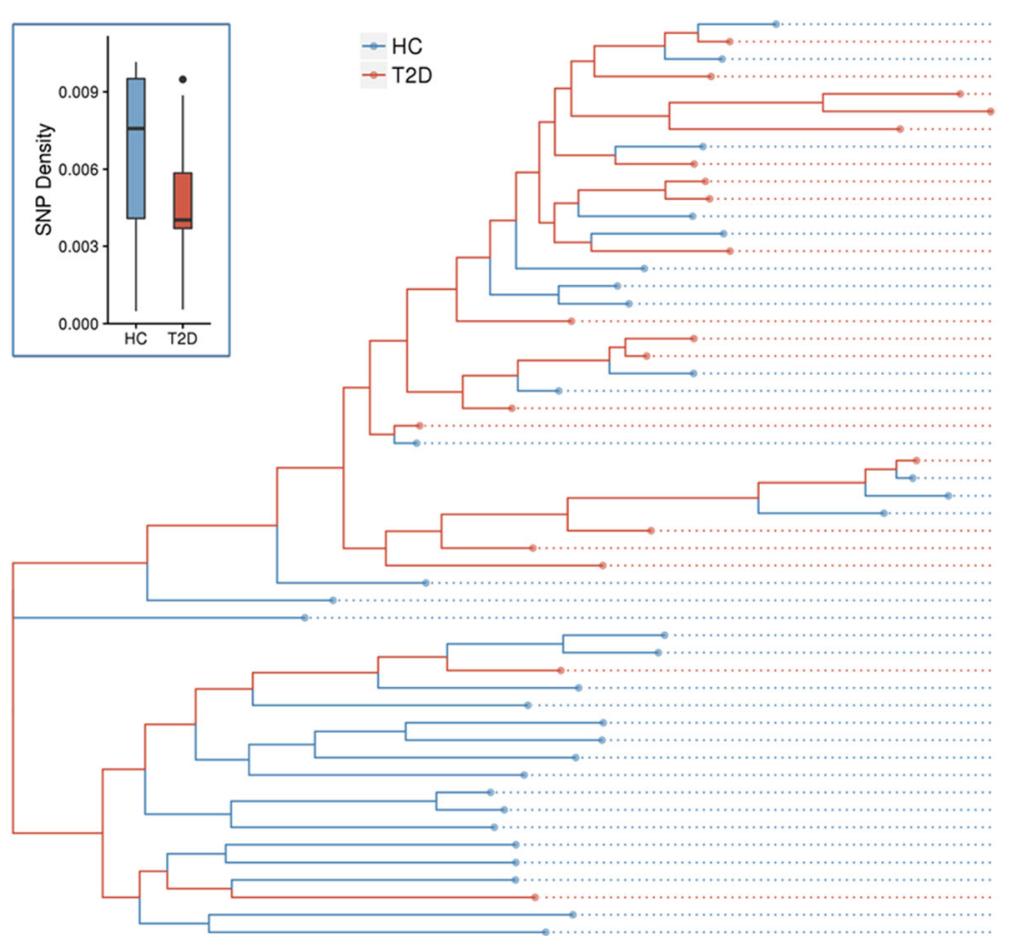

Fig. 1 Phylogenetic tree based on B. coprocola SNPs. Red and blue branches indicate T2D samples and HC (i.e., normal) samples, respectively. The boxplot graph of SNP density for B. coprocola in each sample group is shown in the inset graph

pairwise patristic distance) among T2D individuals' genomes (0.0079) was smaller than that of controls (0.0109) and that determined for the total sample pool (0.0103). We further examined variation distribution of $B$. coprocola under polyclonal scenario. We found that $94.00 \%$ of variations in B. coprocola had a $>0.8 \mathrm{MuAF}$ (Additional file 2: Figure S4). We also built a phylogenetic tree based on SNPs with $>0.8 \mathrm{MuAFs}$ (Additional file 2: Figure S5) and clustered samples by MuAFs of variations (Additional file 2: Figure S6). The results are consistent and imply that T2D patients may share a specific set of B. coprocola strains.

Examination of the SNP distributions of the proteincoding genes of the selected 20 gut bacteria revealed that 51,579 genes in the 20 microbe species had valid coverage with sufficient prevalence (see "Methods" section). Among them, we identified 1300 genes (2.52\%) with significantly differentiated SNP densities between T2D and control samples (Mann-Whitney test, $q<0.05$; Additional file 1: Table S4). All but one of these genes were found in B. coprocola, whose reference genome contains 4291 protein-coding genes. The one gene (EFQ08025.1) not in B. coprocola was from Faecalibacterium $c f$. prausnitzii. With the reference strain of $B$. coprocola DSM 17136, we observed that generally there are more SNPs in the HC group compared to those in the T2D group at the gene level (1268/1300, Additional file 1: Table S4).
To select the genes with the most differentiated SNP distributions, we examined the group bias of each SNP site (Fisher's exact test, $p<0.05$ ) and identified $65 \mathrm{~B}$. coprocola genes with significant enrichment of biased SNPs against the 1300 genes as a background (hypergeometric test, $q<0.05)$. Phylogenetic trees constructed based on the nucleotide sequences of the 65 genes are shown together with their associated gene SNP distributions; the top two genes with the most differentiated SNP distribution are shown in Fig. 2 and the rest of the genes in Additional file 3. Interestingly, we observed clear assemblage of the trees for 49 of these 65 genes into two distinct clusters. The T2D samples enriched in one cluster and the other dominantly consisted of $\mathrm{HC}$ samples (Fisher's exact test, $p<0.05$ ). The most biased gene encodes a glycosyl hydrolase (GenBank accession EDU99824.1) with a biased SNP ratio of 0.59, and the second encodes a response regulator receiver domain protein (GenBank accession EDV02303.1) with a ratio of 0.48 . Both of these genes had a biased distribution of T2D versus HC samples in the two tree clusters (Fisher's exact test, $p<0.05$ ). For example, $88.89 \%$ (16 of 18 ) and $93.75 \%$ (15 of 16) of cluster 1 for the genes EDU99824.1 and EDV02303.1 were from $\mathrm{HCs}$, respectively. Meanwhile, T2D samples were enriched preferentially in the second cluster (18 of 20 for EDU99824.1 and 18 of 19 for EDV02303.1). We further found that $9.08 \%$ of the SNPs in EDU99824.1 and 26.46\% of the SNPs in EDV02303.1 were 
$\mathbf{a}$

EDU99824.1
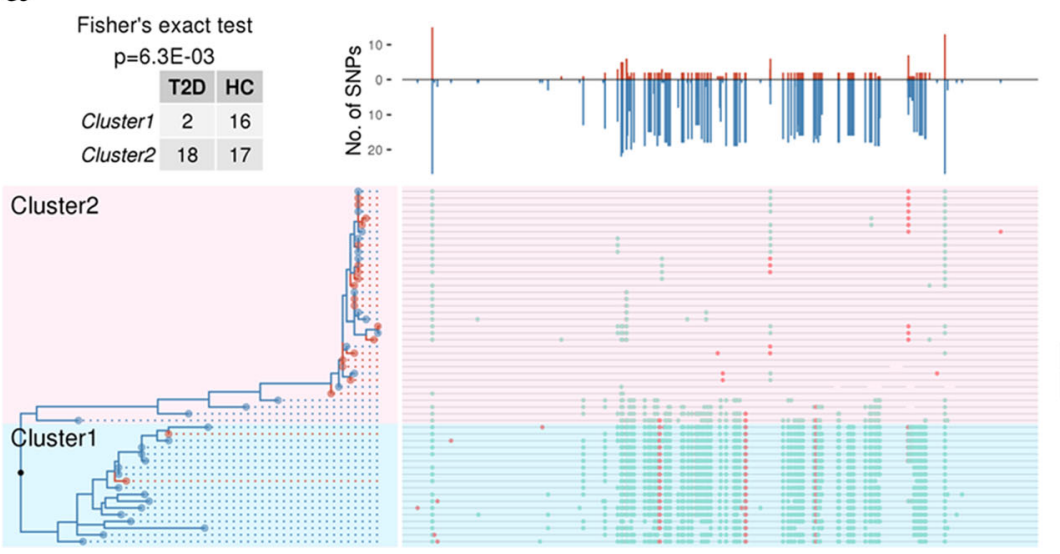

Missense

Silent

b

EDV02303.1
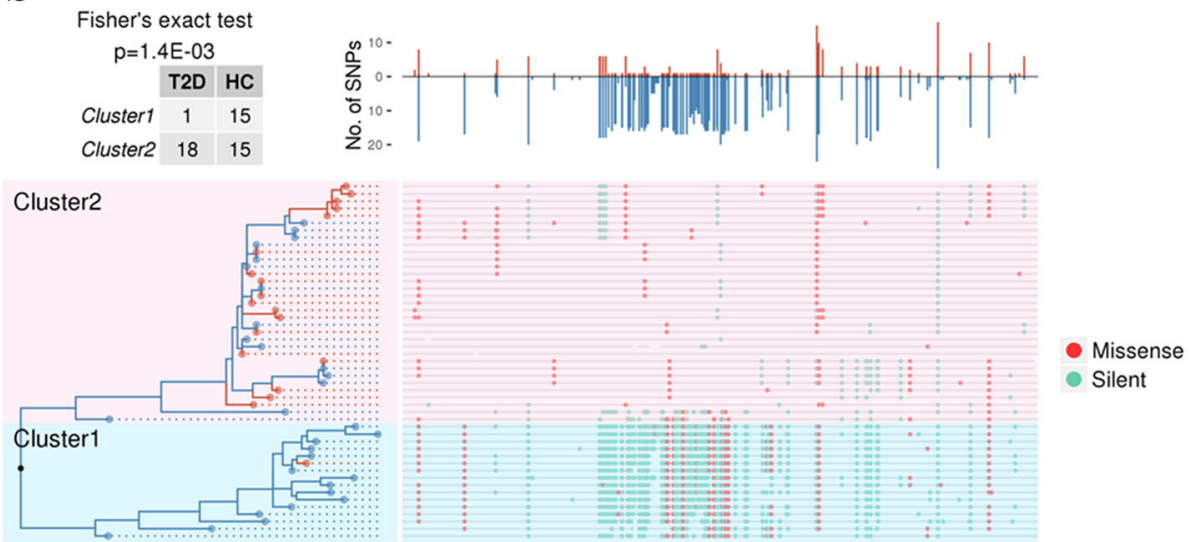

Fig. 2 Phylogenetic trees and SNP distributions of the genes EDU99824.1 (a) and EDV02303.1 (b). Samples in clusters 1 and 2 are indicated by light blue and pink shading, respectively. The lines aligned to tree leaves represent corresponding gene sequences with sufficiently covered reads, with missense (red dot) and silent (green dot) SNPs indicated. The bar graphs above the gene sequences show the number of SNPs found at each site (aligned to each bar) in the T2D group (red bars above the axis) and the HC group (blue bars below the axis). Fisher's exact test results for $2 \times 2$ contingency tables are shown in the upper left of each panel

non-synonymous, and non-synonymous SNPs were differentially distributed between the two clusters (Fisher's exact test, $p=0.02431, p=1.97 \mathrm{E}-09$, respectively). Similar to genome-level analysis under polyclonal scenario, the samples were clustered by MuAFs of each gene. The results also indicated the enrichment of T2D samples in one of the clusters (Additional file 1: Table S5; Additional file 2: Figure S7-S8 for top 2 genes; Additional file 4 for the rest 63 genes). The annotations of the 65 genes with significant enrichment of biased SNP sites between the T2D and control groups are shown in Additional file 1: Table S4. Interestingly, the products of 25 genes $(25 / 65,38.46 \%)$ are annotated to be localized to the cell membrane.

\section{Conclusion}

The present analysis of a Chinese metagenomics dataset revealed that the gut microbiota of T2D patients and $\mathrm{HC}$ individuals had different SNP distributions. The gut microbe species B. coprocola, which had a similar relative abundance between the T2D and $\mathrm{HC}$ groups, exhibited biased SNP distribution at both the genome and the gene level. Our phylogenetic analysis yielded $49 \mathrm{~B}$. coprocola genes that had characteristic SNP distribution in T2D patients. Two of these genes (EDU99824.1 ranked first and EDV02301.1 ranked sixth) encode glycosyl hydrolases. Glycosyl hydrolases of bacteria play vital roles in degradation of cellulose and starch and hence generate sugars. Interestingly, alpha-glucosidase, which is also a glycosyl hydrolase located in the brush border of the small intestine, is an important drug target of T2D. Therefore, it is possible that EDU99824.1 and EDV02301.1 in T2D-related strain and control strain may have different glycosyl hydrolase properties, which is worthwhile to be investigated in the future.

B. coprocola was previously reported to have high SNP density in the gut microbiota [12]. But the correlation between its SNPs and diseases had not been investigated. Our results indicate that a specific set of $B$. coprocola 
strains may be associated with T2D and further suggest that strain-level bacterial colonization of the gut and the potential restorative influence of probiotic supplements should be investigated in T2D therapeutic research.

As is shown, intra-sample variations affect very slightly on our results. However, assuming one strain type per sample may not be general, and intra-sample variations should not be overlooked in strain-level analysis of metagenomics.

\section{Additional files}

Additional file 1: Table S1. The taxonomic lineages of the 356 bacterial species and accessions of their reference genome assemblies. Table S2. Species with significantly different abundance profiles between the T2D and HC groups. Table S3. Detailed descriptive information for 20 bacterial species with sufficiently supporting NGS reads in a sufficient number of samples. Table S4. Summary of the 1300 genes found to have significantly different SNP densities between the T2D and HC groups. The top 65 genes with the most differentiated SNP distributions between the groups are highlighted in light green. Table S5. Intra-tree distances and enrichment analysis based on phylogenetic trees, hierarchical clustering trees, and affinity propagation clustering for the selected 65 genes. Table S6. SNP densities, intra-tree distances, and numbers of the most biased genes under different mutated allele frequency (MuAF) thresholds $(>0.2$, $>0.5$, and $>0.8$ ). (XLS $766 \mathrm{~kb}$ )

Additional file 2: Figure S1. Validation of relative abundance distribution in the T2D and HC groups. Figure S2. Comparisons of SNP densities in 20 gut bacterial species between the T2D and normal groups. Figure S3. Brief flowchart for bioinformatics analysis of metagenomics NGS data at strain level. Figure S4. Distributions of MuAFs at variant sites of $B$. coprocola in T2D (A), HC (B) and all (C) samples with sufficient NGS reads. Figure $S 5$. Phylogenetic tree of $B$. coprocola strains based on variant sites with $>0.8$ MuAFs. Figure S6. Results of AP clustering and hierarchical clustering based on MuAFs of variant sites in B. coprocola. Figure S7. Distributions of MuAFs at variant sites in EDU99824.1 (A) and EDUV02303.1 (B). Figure S8. Results of AP clustering and hierarchical clustering based on MuAFs of variant sites. (PDF $761 \mathrm{~kb}$ )

Additional file 3: Phylogenetic trees of the 63 genes, except the top two, of the 65 genes with the most differentiated SNP distribution. Each tree is in a separate JPG file named by the GenBank accession of the corresponding gene. (RAR $13817 \mathrm{~kb}$ )

Additional file 4: Results of AP clustering, MuAF distributions and hierarchical clustering for 63 genes in the most differentiated SNP distribution except the top two genes. For a given gene, the clusters of AP clustering, MuAF distribution, and hierarchical tree are presented in separated files named by its GenBank accession, within the folders of APclusterTrees, MuAFhistogram, and HlustTrees, respectively. For gene EDV02481.1, samples were failed to be clustered into two clusters, so it has no AP clustering result. (RAR $14532 \mathrm{~kb}$ )

\section{Abbreviations}

GO: Gene Ontology; HC: Healthy control; NCBI: National Center for Biotechnology Information; NGS: Next-generation sequencing; SNP: Singlenucleotide polymorphism; T2D: Type 2 diabetes

\section{Acknowledgements}

We thank Dongsheng Zhao and Xiaolei Wang for their help in computing resources.

\section{Funding}

YC, ZL, SH, JZ, JW, SH, and XY were supported by the China National High Technology Research and Development Program (2014AA020604, X. Ying). $X B$ and $M N$ were supported by the National Natural Science Foundation of China (No. U1435222, X. Bo).

\section{Availability of data and materials}

The metagenomics sequencing data analyzed during this study are included in this published article [3] and the raw Illumina read data are available in the NCBI Sequence Read Archive under accession numbers SRA045646 and SRA050230 (http://www.ncbi.nlm.nih.gov/sra/). Other data supporting the conclusions of this article is included within the article and its additional files.

\section{Authors' contributions}

YC participated in the design of the study, carried out the analysis, and drafted the manuscript. ZL helped to construct the analysis framework. SH helped to perform the statistical analysis. JZ helped to design and plot the figures. JW downloaded the raw data and performed the quality control. NS and XB helped to interpret the results. MN offered guidance on the bioinformatics methods, helped to construct the analysis framework, and drafted and revised the manuscript. XY conceived the study, participated in its design, coordinated and helped to draft the manuscript, and revised the manuscript. All authors read and approved the final version.

\section{Competing interests}

The authors declare that they have no competing interests.

\section{Consent for publication}

Not applicable.

\section{Ethics approval and consent to participate}

The metagenomics sequencing data in this study were generated by this published article [3]. According to the paper, fecal samples were obtained from the volunteers after signing an informed consent form and the sampling procedure was approved by the Ethical Committee for Clinical Research from the Peking University Shenzhen Hospital, Shenzhen Second People's Hospital, and Medical Research Center of Guangdong General Hospital.

Received: 25 July 2016 Accepted: 10 January 2017

Published online: 01 February 2017

\section{References}

1. Turnbaugh PJ, Ley RE, Mahowald MA, Magrini V, Mardis ER, Gordon JI. An obesity-associated gut microbiome with increased capacity for energy harvest. Nature. 2006;444(7122):1027-31. doi:10.1038/nature05414.

2. Larsen N, Vogensen FK, van den Berg FWJ, Nielsen DS, Andreasen AS, Pedersen BK, et al. Gut microbiota in human adults with type 2 diabetes differs from non-diabetic adults. PLoS One. 2010;5:e9085. doi:10.1371/ journal.pone.0009085.

3. Qin J, Li Y, Cai Z, Li S, Zhu J, Zhang F, et al. A metagenome-wide association study of gut microbiota in type 2 diabetes. Nature. 2012;490:5560. doi:10.1038/nature11450.

4. Karlsson FH, Tremaroli V, Nookaew I, Bergström G, Behre CJ, Fagerberg B, et al. Gut metagenome in European women with normal, impaired and diabetic glucose control. Nature. 2013:498:99-103. doi:10.1038/nature12198.

5. Qin N, Yang F, Li A, Prifti E, Chen Y, Shao L, et al. Alterations of the human gut microbiome in liver cirrhosis. Nature. 2014;513:59-64. doi:10.1038/nature13568.

6. Walters WA, Xu Z, Knight R. Meta-analyses of human gut microbes associated with obesity and IBD. FEBS Lett. 2014;588:4223-33. doi:10.1016/j. febslet.2014.09.039.

7. Rather IA, Bajpai VK, KUMAR S, Lim J, Paek WK, Park Y-H. Probiotics and atopic dermatitis: an overview. Front Microbiol. 2016;7. doi:10.3389/fmicb.2016.00507

8. Segal LN, Clemente JC, Tsay J-CJ, Koralov SB, Keller BC, Wu BG et al. Enrichment of the lung microbiome with oral taxa is associated with lung inflammation of a Th17 phenotype. Nature Microbiol. 2016:16031. doi:10. 1038/nmicrobiol.2016.31

9. Bagel S, Hüllen V, Wiedemann B, Heisig P. Impact of gyrA and parC mutations on quinolone resistance, doubling time, and supercoiling degree of Escherichia coli. Antimicrob Agents Chemother. 1999;43:868-75.

10. Sokurenko EV, Chesnokova V, Dykhuizen DE, Ofek I, Wu XR, Krogfelt KA, et al. Pathogenic adaptation of Escherichia coli by natural variation of the FimH adhesin. Proc Natl Acad Sci U S A. 1998;95(15):8922-6.

11. Morowitz MJ, Denef VJ, Costello EK, Thomas BC, Poroyko V, Relman DA, et al. Strain-resolved community genomic analysis of gut microbial colonization in a premature infant. Proc Natl Acad Sci U S A. 2011;108(3): 1128-33. doi:10.1073/pnas.1010992108. 
12. Schloissnig S, Arumugam M, Sunagawa S, Mitreva M, Tap J, Zhu A, et al. Genomic variation landscape of the human gut microbiome. Nature. 2013; 493:45-50. doi:10.1038/nature11711.

13. Greenblum S, Carr R, Borenstein E. Extensive strain-level copy-number variation across human gut microbiome species. Cell. 2015;160(4):583-94. doi:10.1016/j.cell.2014.12.038.

14. Zhu A, Sunagawa S, Mende DR, Bork P. Inter-individual differences in the gene content of human gut bacterial species. Genome Biol. 2015;16:82. doi: 10.1186/s13059-015-0646-9.

15. Donati C, Zolfo M, Albanese D, Tin Truong D, Asnicar F, lebba V et al. Uncovering oral Neisseria tropism and persistence using metagenomic sequencing. Nature Microbiol. 2016:16070. doi:10.1038/nmicrobiol.2016.70

16. Noble D, Mathur R, Dent T, Meads C, Greenhalgh T. Risk models and scores for type 2 diabetes: systematic review. BMJ. 2011;343:d7163. doi:10.1136/bmj.d7163.

17. Bolger AM, Lohse $\mathrm{M}$, Usadel B. Trimmomatic: a flexible trimmer for Illumina sequence data. Bioinformatics. 2014;30(15):2114-20. doi:10.1093/ bioinformatics/btu170.

18. Mitchell A, Bucchini F, Cochrane G, Denise H, ten Hoopen P, Fraser M, et al. EBI metagenomics in 2016-an expanding and evolving resource for the analysis and archiving of metagenomic data. Nucleic Acids Res. 2016;44(D1): D595-603. doi:10.1093/nar/gkv1195.

19. Truong DT, Franzosa EA, Tickle TL, Scholz M, Weingart G, Pasolli E, et al. MetaPhlAn2 for enhanced metagenomic taxonomic profiling. Nat Methods. 2015;12:902-3. doi:10.1038/nmeth.3589.

20. Li H. Aligning sequence reads, clone sequences and assembly contigs with BWA-MEM. arXiv:13033997 [q-bio]. 2013.

21. Storey JD, Tibshirani R. Statistical significance for genomewide studies. Proc Natl Acad Sci U S A. 2003;100(16):9440-5. doi:10.1073/pnas.1530509100.

22. Koboldt $\mathrm{DC}$, Zhang $\mathrm{Q}$, Larson $\mathrm{DE}$, Shen $\mathrm{D}$, McLellan MD, Lin L, et al. VarScan 2: somatic mutation and copy number alteration discovery in cancer by exome sequencing. Genome Res. 2012;22(3):568-76. doi:10.1101/gr.129684. 111.

23. Picard. http://broadinstitute.github.io/picard/. Accessed 20 Jan 2016.

24. Li H, Handsaker B, Wysoker A, Fennell T, Ruan J, Homer N, et al. The sequence alignment/map format and SAMtools. Bioinformatics (Oxford, England). 2009;25:2078-9. doi:10.1093/bioinformatics/btp352.

25. UniProt C. UniProt: a hub for protein information. Nucleic Acids Res. 2015; 43(Database issue):D204-12. doi:10.1093/nar/gku989.

26. Cingolani P, Platts A, le Wang L, Coon M, Nguyen T, Wang L, et al. A program for annotating and predicting the effects of single nucleotide polymorphisms, SnpEff: SNPs in the genome of Drosophila melanogaster strain w1118; iso-2; iso-3. Fly (Austin). 2012;6(2):80-92. doi:10.4161/fly.19695.

27. Stamatakis A. RAXML version 8: a tool for phylogenetic analysis and postanalysis of large phylogenies. Bioinformatics. 2014;30(9):1312-3. doi:10.1093/ bioinformatics/btu033.

28. Kuhner MK, Beerli P, Yamato J, Felsenstein J. Usefulness of single nucleotide polymorphism data for estimating population parameters. Genetics. 2000; 156(1):439-47.

29. Yu G, Smith DK, Zhu H, Guan Y, Lam TT-Y. ggtree: an r package for visualization and annotation of phylogenetic trees with their covariates and other associated data. Methods Ecol Evol. 2016:n/a-n/a. doi:10.1111/2041210X.12628.

30. Bodenhofer U, Kothmeier A, Hochreiter S. APCluster: an R package for affinity propagation clustering. Bioinformatics. 2011;27(17):2463-4. doi:10. 1093/bioinformatics/btr406

31. Shannon P, Markiel A, Ozier O, Baliga NS, Wang JT, Ramage D, et al. Cytoscape: a software environment for integrated models of biomolecular interaction networks. Genome Res. 2003;13(11):2498-504. doi:10.1101/gr. 1239303.

\section{Submit your next manuscript to BioMed Central and we will help you at every step:}

- We accept pre-submission inquiries

- Our selector tool helps you to find the most relevant journal

- We provide round the clock customer support

- Convenient online submission

- Thorough peer review

- Inclusion in PubMed and all major indexing services

- Maximum visibility for your research

Submit your manuscript at www.biomedcentral.com/submit

) Biomed Central 